\title{
HIV-1 Tat Triggers Nuclear Localization of ZO-1 via Rho Signaling and cAMP Response Element-Binding Protein Activation
}

\author{
Yu Zhong, Bei Zhang, Sung Yong Eum, and Michal Toborek \\ Molecular Neuroscience and Vascular Biology Laboratory, Department of Neurosurgery, University of Kentucky Medical Center, Lexington, Kentucky \\ 40536
}

The human immunodeficiency virus (HIV)-specific protein trans-activator of transcription (Tat) can contribute to the dysfunction of brain endothelial cells and HIV trafficking into the brain by disrupting tight junction (TJ) integrity at the blood- brain barrier (BBB) level. Specific TJ proteins, such as zonula occludens (ZO) proteins, localize not only at the cell-cell borders but are also present in the nuclei. The objective of the present study was to evaluate the mechanisms and significance of Tat-induced nuclear localization of ZO-1. Treatment of a brain endothelial cell line (hCMEC/D3 cells) with Tat resulted in a decrease in total levels of ZO-1 but significantly upregulated ZO-1 protein expression in the nuclei. In addition, exposure to Tat stimulated Rho signaling and induced phosphorylation and activity of transcription factor cAMP response element-binding protein (CREB), binding sites that have been identified in the proximal region of the ZO-1 promoter. Interestingly, inhibition of the Rho cascade protected against Tat-induced upregulation of ZO-1 in the nuclei and activation of CREB. Depletion of CREB by infection of cells with specific shRNA lentiviral particles attenuated both Tat-induced Rho signaling and nuclear targeting of ZO-1. A decrease in CREB levels also attenuated Tat-induced endothelial and BBB hyperpermeability as well as transendothelial migration of monocytic cells. The role of CREB in Tat-mediated alterations of ZO-1 was confirmed in brain microvessels in mice with CREB shRNA lentiviral particles injected into the cerebral circulation. The present results indicate the crucial role of Rho signaling and CREB in modulation of nuclear localization of ZO-1 and maintaining the integrity of endothelial monolayers.

\section{Introduction}

Disruption of endothelial tight junctions (TJs) is a feature of several acute and chronic brain disorders, including infection by human immunodeficiency virus-1 (HIV-1) (Dallasta et al., 1999; Boven et al., 2000). Because endothelial cells are not productively infected by HIV-1, several vascular effects of this virus are thought to be mediated by secondary mediators, including the HIV-1-specific protein Tat. Tat is taken up by the cells via insertion into the endosome membranes (Yezid et al., 2009), followed by localization into the nuclei (De Marco et al., 2010). In addition, we demonstrated that Tat, in a dose-dependent manner, can interact with cellular receptors (e.g., vascular endothelial growth factor receptor-2), activate small GTPases, and thus modulate TJ protein expression (András et al., 2005; Zhong et al., 2008, 2010).

\footnotetext{
Received Aug. 19, 2011; revised Oct. 17, 2011; accepted Nov. 5, 2011.

Author contributions: M.T. designed research; Y.Z., B.Z., and S.Y.E. performed research; Y.Z., B.Z., and S.Y.E. analyzed data; Y.Z. and M.T. wrote the paper.

This work was supported by National Institutes of Health Grants MH63022, MH072567, DA027569, and NS39254. The authors declare no competing financial interests.

Correspondence should be addressed to Dr. Michal Toborek, Department of Biochemistry and Molecular Biology, University of Miami School of Medicine, R. Bunn Gautier Building, Room 517, 1011 NW 15th Street, Miami, FL 33136. E-mail:mtoborek@med.miami.edu.

S. Y. Eum and M. Toborek's present address: Department of Biochemistry and Molecular Biology, University of Miami School of Medicine, Miami, FL 33136.

DOI:10.1523/JNEUROSCI.4266-11.2012

Copyright $\odot 2012$ the authors $\quad 0270-6474 / 12 / 320143-08 \$ 15.00 / 0$
}

Tetraspan transmembrane proteins (e.g., occludin and claudins) constitute the backbone of TJ strands, which are associated with the actin cytoskeleton via several adaptor molecules, such as zonula occludens-1 (ZO-1), ZO-2, and ZO-3. ZO proteins bind directly to F-actin and other proteins that regulate cytoskeletal dynamics (Schneeberger and Lynch, 2004). Indeed, depletion of ZO-1 alters the dynamics of F-actin redistribution after a calcium switch (McNeil et al., 2006) in Madin-Darby canine kidney (MDCK) cells. However, the cellular role of ZO proteins appears to be much more complex than scaffolding functions at the junctional sites and also involves intracellular signaling and gene expression. For example, ZO-2 was demonstrated to suppress gene transcription by binding to the AP-1 transcription factor protein complex (Betanzos et al., 2004; Bauer et al., 2010).

It has been recognized that the Rho signaling pathway has modulatory effects on $\mathrm{TJ}$ integrity and endothelial barrier properties. For example, inhibition of Rho lowered permeability of bovine microvascular endothelium (Carbajal and Schaeffer, 1999) and reduced baseline permeability of both post-capillary venules in vivo and different cultured microvascular endothelial cells in vitro (Adamson et al., 2002; Baumer et al., 2008). Conversely, activation of RhoA downstream of several permeability-increasing mediators, such as thrombin or TNF- $\alpha$, contributed to increased endothelial permeability (Wojciak-Stothard and Ridley, 2002). Inhibition of the Rho pathway in brain microvascular endothelial cells also protected against monocyte-induced phosphorylation of occlu- 
din and claudin- 5 and diminished transendothelial migration of HIV-1-infected monocytes (Persidsky et al., 2006).

The cAMP response element-binding protein (CREB) is a transcription factor activated by phosphorylation on Ser ${ }^{133}$. Recent reports provide evidence that CREB can be involved in regulation of epithelial junction function. Indeed, the transcriptional repression of ZO-1 by JunD appears to be mediated through a CREB-binding site within the proximal region of the ZO-1 promoter. In the present study, we focused on the signaling and transcriptional mechanisms that regulate nuclear targeting of ZO-1 by HIV-1 protein Tat. We demonstrate that exposure to Tat induces nuclear localization via activation of Rho signaling and activation of CREB binding to a ZO-1 promoter in brain endothelial cells. Importantly, depletion of CREB protected both against Tat-induced alterations of ZO-1 levels and disruption of endothelial integrity.

\section{Materials and Methods}

Cell cultures, infection with shRNA lentiviral particles, and Tat treatment. Human brain cortical microvessel endothelial cells (hCMEC/D3) (Weksler et al., 2005) were cultured as described previously (Zhong et al., 2008). Before exposure to treatment factors, hCMEC/D3 cells were incubated in EBM-2 medium without FBS and EGM-2 SingleQuots (Lonza) for $24 \mathrm{~h}$. For transendothelial migration studies, cocultures of hCMEC/D3 with human monocytic (THP-1) cells were used. THP-1 cells were cultured in RPMI-1640 medium supplemented with 10\% FBS.

For specific experiments, hCMEC/D3 cells were infected with shRNA lentiviral particles containing target-specific constructs encoding for CREB-1 (h) shRNA and control shRNA (both from Santa Cruz Biotechnology) using the instructions of the manufacturer. This procedure resulted in $\sim 50 \%$ decrease in CREB expression. Stable cell lines were selected in a medium containing $10 \mu \mathrm{g} / \mathrm{ml}$ puromycin and maintained in the presence of puromycin in EBM-2 completed medium. The cells with decreased CREB expression were fully viable.

HIV-1 Tat protein was purified as described by Ma and Nath (1997). To determine specificity of Tat-induced effects, Tat solutions were immunoabsorbed using anti-Tat antibody conjugated to protein-A/G plusagarose (Santa Cruz Biotechnology), followed by centrifugation to remove precipitated proteins. This antibody-absorption mixture was named AA-Tat. Another control form of Tat was heat-inactivated Tat (H-Tat) obtained by heating the protein at $90^{\circ} \mathrm{C}$ for $1 \mathrm{~h}$, which inactivates biological potentials of proteins but does not affect activity of endotoxins such as LPS.

Adenovirus infection. Recombinant adenovirus encoding for green fluorescent protein (GFP) and the Rho inhibitor Clostridium botulinum exoenzyme C3 transferase, the exoenzyme that specifically ribosylates and inhibits the function Rho gene, was produced as described previously (Hirai et al., 2007) and named the C3 adenovirus. A separate adenovirus vector expressing GFP without exoenzyme C3 transferase was constructed as a control (control adenovirus). The viruses were amplified by repeated infection into human embryonic kidney 293 cells. Media containing recombinant adenoviruses were collected and stored at $-80^{\circ} \mathrm{C}$. hCMEC/D3 cells were infected with recombinant adenoviruses at a multiplicity of infection of 200 in a 6 or $10 \mathrm{~cm}$ culture dish. Infection with adenoviruses resulted in efficiency close to $100 \%$ as determined by monitoring GFP signal under a fluorescent microscope.

Cell fractionation, immunoblotting, and electrophoretic mobility shift assay. Cell fractions were obtained using a nuclear and cytoplasmic extraction reagents kit from Thermo Fisher Scientific and analyzed by Western blotting using standard approaches. The density of the bands was calculated with the Scion Image program. CREB, phosphorylated CREB (p-CREB) $\left(\operatorname{Ser}^{133}\right)$, phospho-myosin light chain 2 (MLC) $\left(\operatorname{Ser}^{19}\right)$, and MLC2 antibodies were purchased from Cell Signaling Technologies. RhoA, vascular endothelial (VE)-cadherin, and all secondary antibodies were from Santa Cruz Biotechnology. ZO-1 and claudin-5 antibodies were from Invitrogen. MLC kinase (MYLK) and anti-actin antibodies were from Sigma.
For electrophoretic mobility shift assay (EMSA), synthetic oligonucleotides were produced and 5-biotinylated by Integrated DNA Technologies. The sequence for the wild-type CREB was 5'CCG GGT GCC CAT GAC GTC ATC ACC AGT TTC AGC 3' and for the mutant CREB was 5'CCG GGT GCC CAT GAC GTC TAC ACC AGT TTC AGC 3'. Binding reactions were performed using a LightShift Chemiluminescent EMSA kit (Pierce) following the instructions of the manufacturer.

Integrity of $h C M E C / D 3$ monolayers and transendothelial migration. For endothelial permeability and transendothelial migration measurements, cells were cultured on 0.4 and $3.0 \mu \mathrm{m}$ Transwell inserts, respectively, until confluency. The cells were treated with $100 \mathrm{~nm} \mathrm{HIV-1} \mathrm{Tat} \mathrm{and}$ control Tat (AA-Tat or H-Tat) for $20 \mathrm{~h}$. Endothelial permeability was assessed by the flux of FITC-dextran 20 (molecular weight $20 \mathrm{kDa} ; 1$ $\mathrm{mg} / \mathrm{ml}$; Sigma) across endothelial monolayers. For transmigration assay, treated cells growing on the $3.0 \mu \mathrm{m}$ membrane inserts were placed in the wells containing serum-free endothelial basal medium with added CCL-2 $(50 \mathrm{ng} / \mathrm{ml})$ as chemoattractant. Monocytic THP-1 cells were stained with calcein $\mathrm{AM}(5 \mu \mathrm{M}$, for $25 \mathrm{~min})$ and added in the amount of $5 \times 10^{5}$ on top of hCMEC/D3 monolayers for $4 \mathrm{~h}$ at $37^{\circ} \mathrm{C}$. Florescence was measured as a marker of THP-1 cell migration at $485 \mathrm{~nm}$ excitation and $530 \mathrm{~nm}$ emission wavelengths.

Surgical procedures, animal treatment, and brain microvessel analyses. All experimental procedures and protocols were reviewed and approved by the Animal Care and Use Committee of the University of Kentucky. C57BL/6 mice (10-week-old males) were injected with $40 \mu$ l of CREB- 1 (m) shRNA or control shRNA lentiviral particles $\left(1 \times 10^{6}\right.$ infectious units; both from Santa Cruz Biotechnology) into the internal carotid artery (ICA) using a surgical technique described by our research group (Chen et al., 2009). Forty-eight hours after lentiviral particle administration, Tat (50 $\mu \mathrm{g} /$ mouse) was injected into the ICA. Brain microvessels were isolated $24 \mathrm{~h}$ after Tat injection as described previously (Seelbach et al., 2010). The obtained microvessels were either smeared on slides for fluorescence microscopy analysis or resuspended in $0.5 \mathrm{ml}$ of $6 \mathrm{M}$ urea lysis buffer containing $6 \mathrm{~m}$ urea, $0.1 \%$ Triton X-100, $10 \mathrm{~mm}$ Tris, $\mathrm{pH}$ 8.0, $5 \mathrm{~mm} \mathrm{MgCl}_{2}, 5 \mathrm{~mm}$ EDTA, and $150 \mathrm{~mm} \mathrm{NaCl}$ with Complete Protease Inhibitor for Western blot analyses. Integrity of the blood-brain barrier (BBB) was measured $24 \mathrm{~h}$ after Tat injection as described previously (Chen et al., 2009) by injecting $100 \mu \mathrm{l}$ of $2 \%$ sodium fluorescein (NaF) into the ICA. Fifteen minutes after $\mathrm{NaF}$ injection, permeability across the $\mathrm{BBB}$ was calculated as the ratio of fluorescence in the brain tissue to plasma.

Statistical analysis. Each experiment was repeated a minimum of three times. Data were expressed as mean \pm SD. Statistical analysis was determined by using $t$ test for two groups and one-way ANOVA to compare mean responses among the treatments. Statistical probability of $p<0.05$ was considered significant.

\section{Results}

Rho signaling differentially regulates Tat-induced alterations of total and nuclear $\mathrm{ZO}-1$ expression

hCMEC/D3 cultures were treated with Tat for $15 \mathrm{~h}$, and ZO- 1 protein expression was evaluated in total cell lysates and nuclear extracts by Western blotting. As indicated in Figure $1 \mathrm{~A}$, treatment with Tat significantly decreased total ZO-1 levels in cells that were mock infected or infected with a control adenovirus. However, inhibition of Rho by adenovirus expressing exoenzyme C3 transferase (C3 adenovirus) markedly protected against Tatinduced alterations of ZO-1 levels. The observed effects were specific because infection with control adenovirus did not affect the basal levels of ZO-1. In addition, exposure to AA-Tat did not affect ZO-1 levels. Expression of actin (housekeeping protein) also was not affected by the infection procedure.

To further determine the relationship between the Rho signaling and ZO-1 expression, ROCK (the Rho downstream kinase) activity was inhibited by a 30 min pretreatment with Y27632 (4-[( $1 R)$-1-aminoethyl]- $N$-pyridin-4-yl-cyclohexane-1-carboxamide) $(5 \mu \mathrm{M})$. Similar to $\mathrm{C} 3$ adenovirus, treatment with Y27632 
A
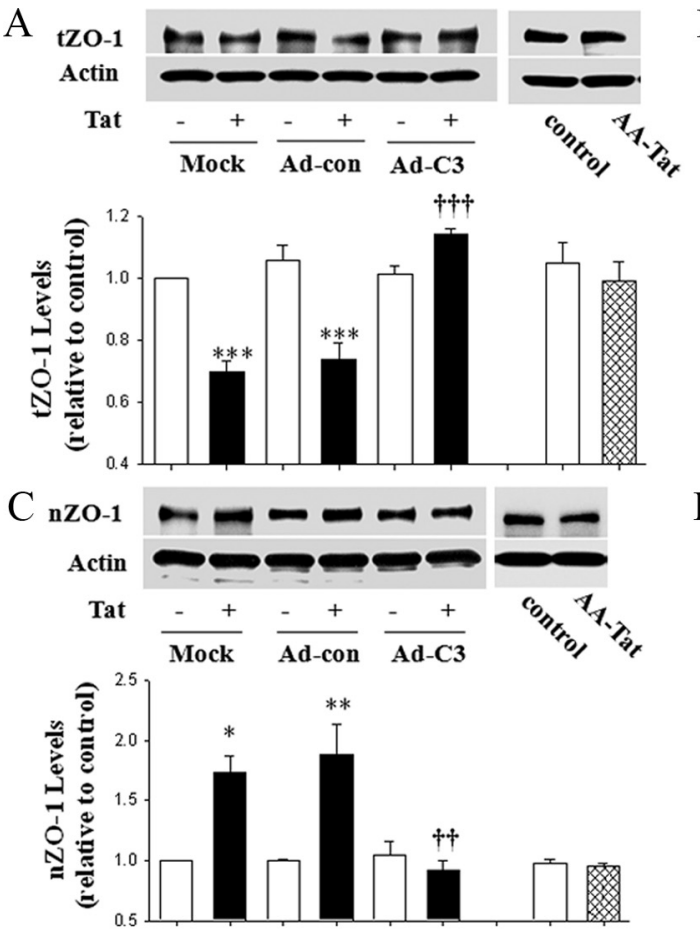

B
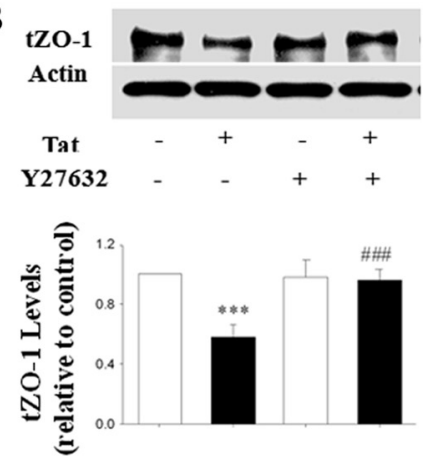

D

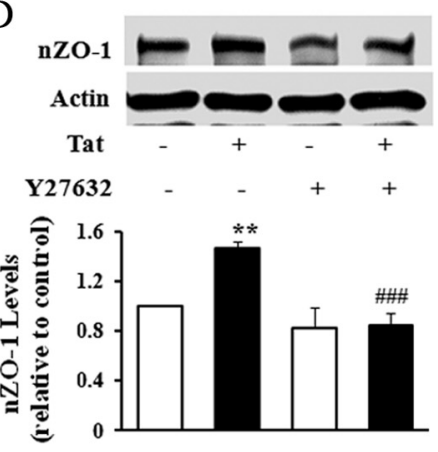

Figure 1. Rho signaling modulates Tat-induced alterations of total and nuclear expression of Z0-1. $A, C, h C M E C / D 3$ cultures at $\sim 50 \%$ confluence were infected with C3 adenovirus (Ad-C3) or control adenovirus (Ad-con). Two days after infection, cells were exposed to $100 \mathrm{~nm}$ Tat and Z0-1 protein levels were measured by immunoblotting in total cell lysates after treatment for $15 \mathrm{~h} \mathrm{(A)}$ or nuclear extracts after treatment for $1 \mathrm{~h}$ (C). For control experiments, the mock- or adenovirus-infected cells were incubated with $100 \mathrm{~nm}$ Tat for 15 h. B, D, Confluent hCMEC/D3 cultures were pretreated with Y27632 (5 $\mu \mathrm{m}$; ROCK inhibitor) for $30 \mathrm{~min}$ and then exposed to Tat (100 nM). 20-1 levels were detected in whole-cell lysates after a $15 \mathrm{~h}$ exposure $(\boldsymbol{B})$ or in nuclear extracts after a $1 \mathrm{~h}$ exposure $(\boldsymbol{C})$ by immunoblotting. 20-1 expression was normalized to actin levels. The blots reflect representative data from three different experiments (mean \pm SD), and the bar graphs are quantified data from these experiments. ${ }^{*} p<0.05,{ }^{* *} p<0.01$ or ${ }^{* * *} p<0.001$, compared with the respective control (vehicle-treated cells). ${ }^{\dagger+} p<0.01$ or ${ }^{\mathrm{tt \dagger}} p<0.001$, data in cultures exposed to Tat plus C 3 adenovirus are significantly different from Tat plus mock infection or Tat plus control adenovirus. ${ }^{\# \#} p<0.001$, data in cultures exposed to Tat plus Y27632 are significantly different from those in cultures exposed to Tat alone.
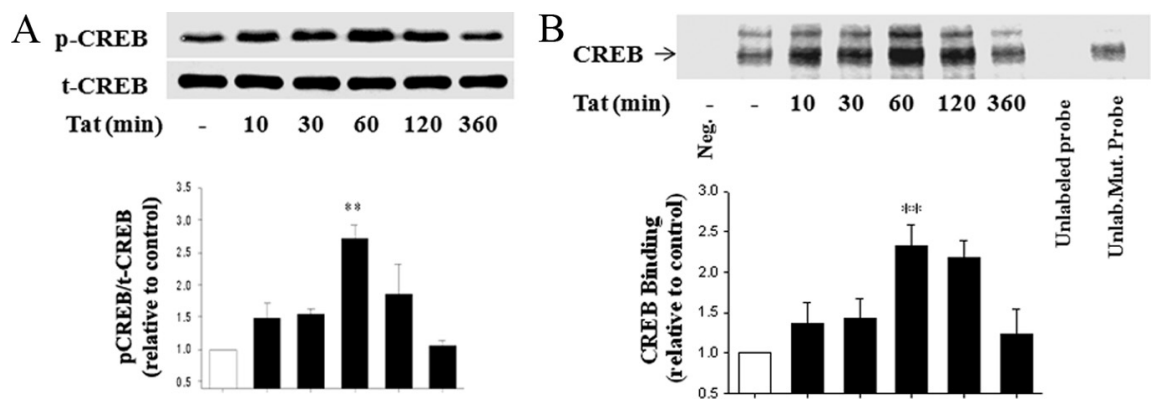

Figure 2. Tat stimulates CREB phosphorylation and binding activity. Time-dependent effects of Tat on phosphorylation of CREB $(\boldsymbol{A})$ and DNA binding activity $(\boldsymbol{B})$. Cells were exposed to $100 \mathrm{~nm}$ Tat for the indicated time points. $\mathbf{A}, \mathrm{p}$-CREB was determined by immunoblotting with specific anti-phospho-CREB $\left(\right.$ Ser $^{133}$ ) antibody, and the levels were normalized to t-CREB levels. B, CREB DNA binding activity was analyzed by EMSA. Competition study was performed by adding 100 -fold molar excess of unlabeled wild-type CREB probe (the lane before last) or unlabeled mutated probe (last lane). First lane (Neg), nuclear protein was not added to binding reaction. The images are representative blots, and the bar graphs are pooled densitometry data from three experiments (mean \pm SD). ${ }^{* *} p<0.01$, compared with vehicle-treated cells.

significantly protected against a Tat-induced decrease of ZO-1 protein levels in total cell extracts (Fig. $1 B$ ).

We next evaluated the effects of Tat and Rho signaling on ZO-1 levels in the nuclei of hCMEC/D3 cells. Although Tat treatment decreased the total level of ZO-1, it exerted the opposite effect on ZO-1 in nuclear fractions. As shown in Figure $1 C$, a $1 \mathrm{~h}$ exposure to $100 \mathrm{~nm}$ Tat (but not AA-Tat) significantly increased

ZO-1 expression in nuclear fractions of mock or control adenovirus-infected hCMEC/D3 cells. These effects were completely prevented by the inhibition of Rho by $\mathrm{C} 3$ adenovirus (Fig. 1C) or inhibition of ROCK by a 30 min pretreatment with Y27632 (5 $\mu \mathrm{M})$ (Fig. 1D). It is now well established that actin is present in the nuclei (Pederson, 2008) and its cytoplasmic and nuclear pools are linked (Hofmann, 2009). Therefore, the levels of nuclear actin were detected as internal controls.

To determine the specificity of ZO-1 responses, we determined the effects of Tat on cellular levels of VE-cadherin. hCMEC/ D3 cells were characterized by strong cytoplasmic VE-cadherin expression, with virtually no presence in the nuclei. Tat exposure did not affect cellular VE-cadherin levels nor did it induce nuclear translocation of this protein (data not shown).

\section{Tat simulates CREB phosphorylation} and binding activity

Because CREB might be involved in transcriptional regulation of ZO-1 expression (Chen et al., 2008), we next evaluated the influence of Tat on CREB activation. Treatment of hCMEC/D3 cultures with Tat resulted in a time-dependent increase in p-CREB as determined by Western blotting. The most pronounced $(\sim 2.7-$ fold) induction of p-CREB levels was observed after a $1 \mathrm{~h}$ exposure to $100 \mathrm{~nm}$ Tat. Total CREB ( $\mathrm{t}$-CREB) levels did not change in response to Tat (Fig. 2A). We also evaluated CREB DNA binding activity using biotin-labeled oligonucleotide probe with the sequence specific for the CREB binding sites on ZO-1 promoter. Exposure to $100 \mathrm{~nm}$ Tat induced a timedependent increase in CREB binding with the maximum effect at $1 \mathrm{~h}$ of treatment. The specificity of this effect was confirmed by a competition assay using a 100fold molar excess of unlabeled wild-type or unlabeled mutated oligonucleotide probes. The CREB binding signal disappeared in the presence of an excess of the CREB wild-type competitor but was unaffected in the presence of a mutated competitor (Fig. 2B).

Tat induces the interactions between the Rho and CREB pathways

To evaluate a possible interaction between Rho signaling and CREB activity, a series of immunoprecipitation studies was performed using anti-CREB antibody. As indicated in Figure $3 A$, CREB immunoprecipitated with p-CREB, RhoA, MLC, and MYLK, a downstream kinase from Rho. Importantly, treatment with $100 \mathrm{~nm}$ Tat for $1 \mathrm{~h}$ markedly increased these interactions, reflecting increased phosphorylation of CREB and activation of the Rho pathway. 

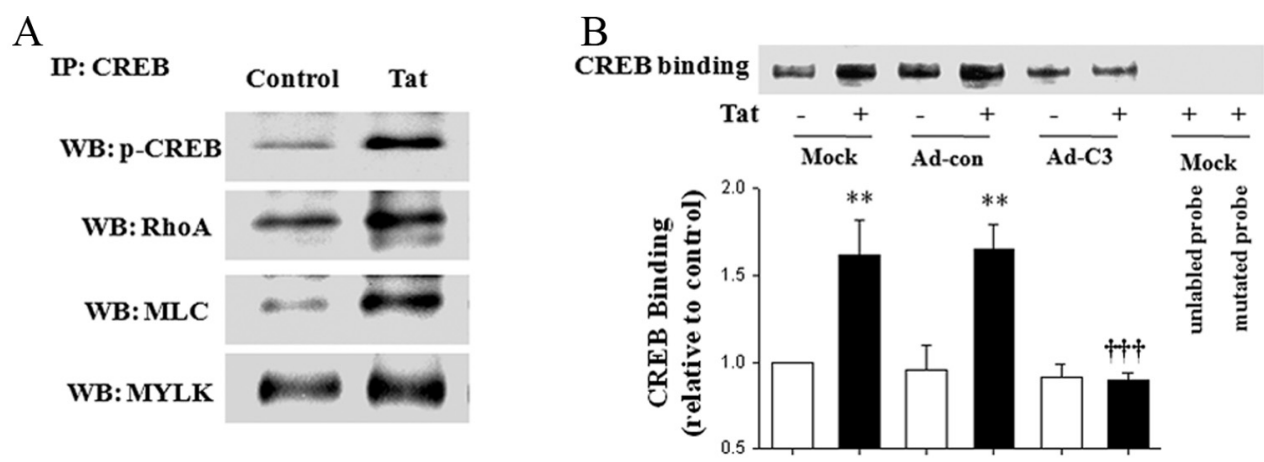

$\mathrm{C}$
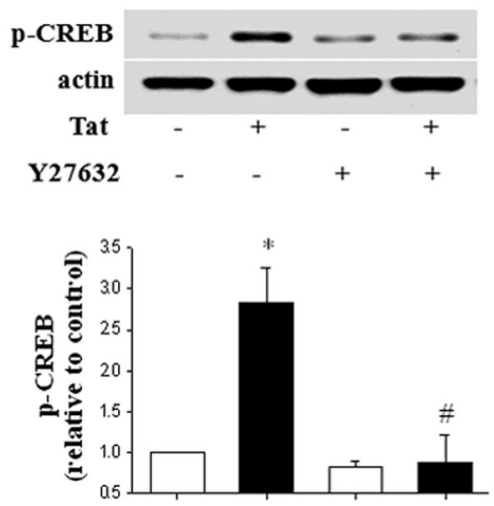

$\mathrm{D}$
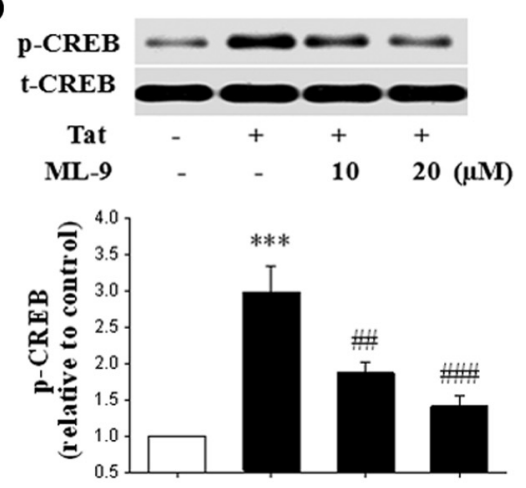

E p-MLC
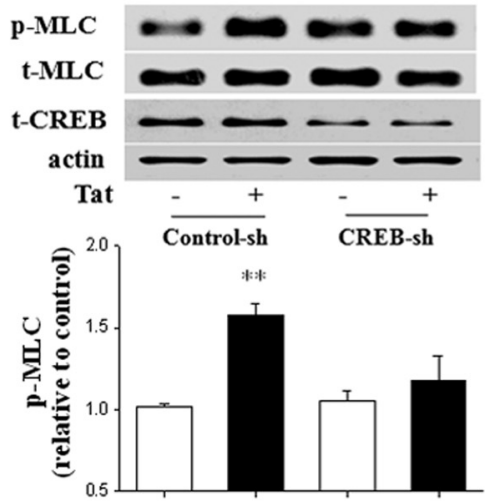

Figure 3. Tat-induced Rho signaling and CREB activation are cross-dependent. $A$, CREB coimmunoprecipitates with Rho kinases. Confluent hCMEC/D3 cells were treated with $100 \mathrm{~nm}$ Tat for $1 \mathrm{~h}$, and cell lysates containing the same amounts of proteins were immunoprecipitated using anti-CREB antibody, followed by determination of p-CREB, RhoA, MLC, and MYLK by immunoblotting. WB, Western blot. B, hCMEC/D3 cells were infected with C3 or control adenovirus (Ad-C3 and Ad-con, respectively) as in Figure 1 A. Then, the cells were treated with $100 \mathrm{~nm}$ Tat for $1 \mathrm{~h}$, and nuclear proteins were analyzed for CREB DNA binding by EMSA. Specificity of the binding reaction was determined by adding an excess of unlabeled probe (the lane before last) or mutated probe (last lane). $C$, Subconfluent hCMEC/D3 cells were pretreated with Y27632 (5 $\mu \mathrm{M}$ ) for $30 \mathrm{~min}$ and then exposed to Tat (100 $\mathrm{nm}$ ) for $1 \mathrm{~h}$. CREB phosphorylation was detected by immunoblotting as in Figure $2 \mathrm{~A}$ and normalized to actin levels. D, Subconfluent hCMEC/D3 cells were pretreated with the indicated doses of ML-9 (MLCK inhibitor) for $30 \mathrm{~min}$ and then exposed to Tat (100 nM) for $1 \mathrm{~h}$. CREB phosphorylation was detected by immunoblotting and normalized to t-CREB levels. E, Cellular CREB was depleted by infecting hCMEC/D3 cells with specific CREB shRNA (CREB-sh). Control cultures were infected with control shRNA lentiviral particles (Control-sh). The cells were then treated with Tat (100 nM) for $1 \mathrm{~h}$, followed by determination of total and phosphorylated MLC by immunoblotting. t-CREB was determined to indicate cellular CREB depletion. The blots reflect representative data from four to five different experiments, and the bar graphs represent quantified results (mean \pm SD) from these experiments as analyzed by densitometry. ${ }^{*} p<0.05$ or ${ }^{* *} p<0.01$, compared with the respective control (vehicle-treated cells). ${ }^{t+t} p<0.001$, data in cultures exposed to Tat plus C 3 adenovirus are significantly different from Tat plus mock infection or Tat plus control adenovirus. ${ }^{\#} p<0.05,{ }^{\# \#} p<0.01$, or ${ }^{\# \# ~} p<0.001$, data in cultures treated with Tat plus Y27632 or Tat plus ML-9 are significantly different from those in cultures treated with Tat alone.

We then evaluated the involvement of Rho signaling in Tatinduced CREB DNA binding by blocking Rho pathways via infecting hCMEC/D3 cells with C3 adenovirus. Control cells were mock infected or infected with the control adenovirus. The cultures were then exposed to Tat for $1 \mathrm{~h}$ and CREB binding was determined in the nuclear proteins. As indicated in Figure $3 B$, treatment with Tat significantly increased CREB binding activity in control cultures. However, this effect was markedly attenuated by infection with $\mathrm{C} 3$ adenovirus. Tat-induced CREB binding was also prevented by pretreatment with ROCK inhibitor Y27632 (5 $\mu \mathrm{M}$ for $30 \mathrm{~min}$ ) (data not shown). Finally, pretreatment with Y27632 or with MYLK inhibitor ML-9 [1(5-chloronaphthalene-1-sulfonyl)-1H-hexahydro-1,4-diazepine] completely abolished Tat-mediated phosphorylation of CREB (Fig. $3 C, D$, respectively).

Next, we studied the influence of CREB on Rho signaling. Cellular CREB levels were depleted by infecting hCMEC/D3 cells with specific CREB shRNA lentiviral particles, whereas control cultures were infected with control shRNA lentivirus. This procedure resulted in an $\sim 50 \%$ decrease in cellular CREB (data not shown). Importantly, CREB depletion inhibited Tatmediated phosphorylation of MLC (Fig. 3E), indicating the dependency of the Rho pathway on CREB levels. Thus, the results in Figures 3 and 4 indicate that the Rho cascade closely interacted with CREB, resulting in cross-activation of these signaling pathways.

\section{CREB activity is required for Tat-induced nuclear targeting of ZO-1}

Because inhibition of the Rho pathway protected against Tat-induced nuclear localization of ZO-1 (Fig. 1C,D) and Rho closely interacts with CREB (Fig. 3), we next addressed the role of CREB in Tatinduced elevation of nuclear ZO- 1 levels. The experiments were performed on hCMEC/D3 cells in which CREB was depleted by infection with specific CREB shRNA lentiviral particles. Consistent with the results shown in Figure 1, a decrease in total ZO-1 levels was observed in control cultures exposed to $100 \mathrm{~nm}$ Tat for $15 \mathrm{~h}$ (Fig. 4A); however, a $1 \mathrm{~h}$ Tat treatment caused an increase in nuclear ZO-1 (Fig. 4B). Importantly, depletion of CREB fully abolished these effects in both total cell lysates and nuclear fraction.

To further link these findings with CREB activation, we determined the effects of Tat on CREB phosphorylation and binding activity in control and CREB-depleted hCMEC/D3 cells. Using conditions established in experiments reflected in Figure 3, cells were treated with $100 \mathrm{~nm}$ Tat for $1 \mathrm{~h}$. Tat-induced phosphorylation of CREB was completely inhibited in CREB-depleted cells (Fig. 4C). Similarly, Tat-stimulated CREB DNA binding activity 
A
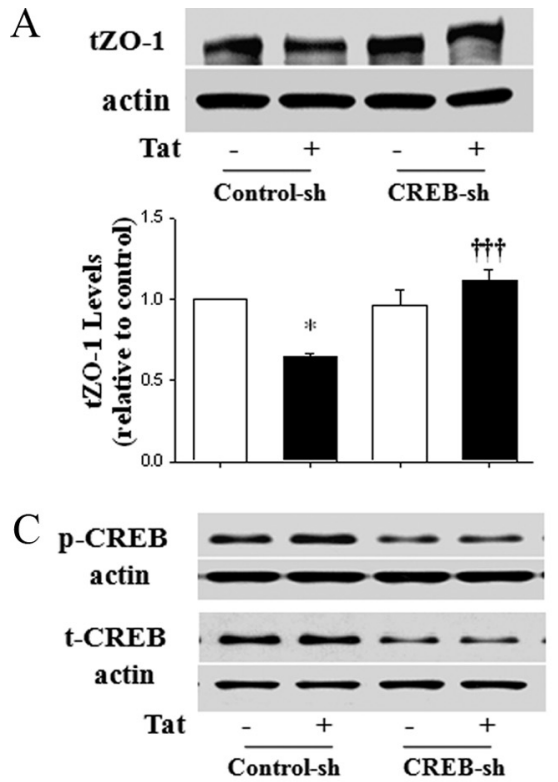

$\mathrm{D}$
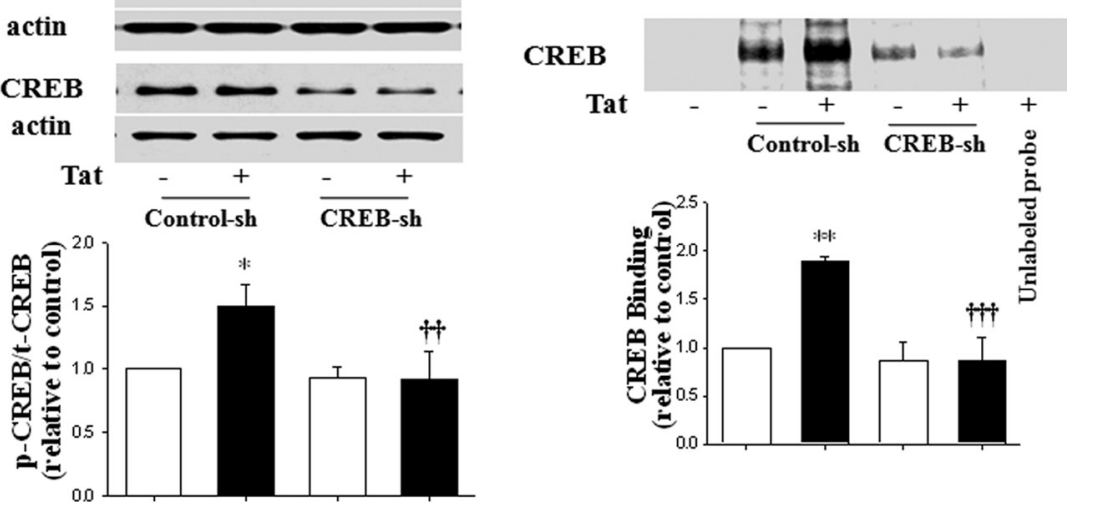

Figure 4. CREB activity is required for Tat-induced nuclear targeting of Z0-1. Cellular CREB was depleted as in Figure 3D, followed by treatment with Tat $(100 \mathrm{~nm})$ for $15 \mathrm{~h}(\boldsymbol{A})$ or $1 \mathrm{~h}(\boldsymbol{B}-\boldsymbol{D})$. 20-1 was determined in total cell lysates $(\boldsymbol{A})$ and in nuclear fraction $(\boldsymbol{B})$. The levels of $\mathrm{p}$-CREB were determined by immunoblotting in total cell lysates $(\boldsymbol{C})$ and CREB DNA binding activity was measured by EMSA (D). Specificity of DNA binding was determined by adding a 100-fold excess of unlabeled probe. Z0-1 expression was normalized to actin levels, and p-CREB expression was normalized to t-CREB levels. The blots reflect representative data from three to four different experiments, and the bar graphs represent quantified results (mean \pm SD) from these experiments as analyzed by densitometry. ${ }^{*} p<0.05$ or ${ }^{* *} p<0.01$, compared with the respective control (vehicle-treated cells). ${ }^{\dagger} p<0.05$, ${ }^{+\dagger} p<0.01$, or ${ }^{{ }^{t+}} p<0.001$, data in cultures exposed to Tat plus CREB shRNA lentiviral particles are significantly different compared with Tat plus control shRNA.

to oligonucleotide probes with the binding sequences specific for ZO-1 promoter was abolished in nuclear fractions of CREBdepleted hCMEC/D3 cells (Fig. 4D).

Tat-induced alterations of ZO-1 expression are attenuated by CREB depletion in an animal model

The role of CREB in Tat-induced alteration of ZO-1 expression was evaluated in mouse brain capillaries. Administration of Tat to control shRNA-injected mice resulted in a decrease in immunoreactivity and fragmentation of staining continuity for ZO-1. Importantly, these effects were abolished in CREB-depleted brain microvessels (Fig. 5A). Densitometric quantification of Western blots confirmed that Tat-mediated diminished levels of ZO-1 protein were statistically significant in brain microvessels isolated from CREB-depleted mice compared with controls (Fig. 5B). The blots in Figure $5 B$ confirm that injections with CREB shRNA lentiviral particles into the ICA effectively depleted CREB in brain microvessels. In addition, CREB depletion protected against Tat-induced disruption of the BBB integrity (Fig. $5 C$ ).

CREB depletion protects again Tat-induced disruption of the endothelial barrier function

The final series of experiments determined the effects of CREB depletion on the functional status of endothelial monolayers. As indicated in Figure 6A, treatment with Tat (but not with AA-Tat and $\mathrm{H}$-Tat) disrupted the endothelial barrier function as illustrated by increased FITC-dextran 20 flux across cultured hCMEC/D3 monolayers. Importantly, CREB depletion by infecting the cells with CREB shRNA lentiviral particles significantly protected against these effects, mimicking in vivo results presented in Figure 5C. Similarly, CREB depletion completely protected against Tat-induced adhesion of monocytic cells to endothelial monolayers (Fig. 6B).

\section{Discussion}

Disruption of the integrity of the brain endothelium is well documented in HIV-1 infection. Clinical studies have reported the absence or fragmentation of ZO- 1 and occludin in HIV-positive patients with encephalitis or HIV-1-associated dementia (Dallasta et al., 1999; Boven et al., 2000; Eugenin et al., 2006). To confirm the role of TJs in HIV-1-associated brain pathology, it has been shown that monocyte migration occurs between endothelial-cell borders and TJ disruption, correlated with the intensity of monocyte infiltration in human brain tissue with HIV encephalitis (Persidsky et al., 2006). In addition, monocyte infiltration has been associated with the disruption of ZO-1 immunoreactivity in HIV-positive patients (Boven et al., 2000). HIV-1 protein Tat has been shown to alter the expression of several TJ proteins, including occludin, claudin-5, ZO-1, and ZO-2 (Zhong et al., 2008). However, there is no information available on the effects of HIV-1 and/or HIV-1-specific proteins on nuclear localization of TJ proteins and on the regulatory mechanisms that control such localization.

Although HIV-1 has been shown to decrease cellular expression of ZO-1 in vitro and in an animal model (Luabeya et al., 2000; Huang et al., 2009), novel results of the present study indicate that this effect is accompanied by a significant increase in nuclear levels of ZO-1. Moreover, inhibition of Rho signaling by C3 transferase or Y27632 protected against Tat-mediated alterations of nuclear localization of ZO-1. These results are in agreement with our previous report that Tat is a potent stimulator of Rho signaling in brain endothelial cells (Zhong et al., 2010). In addition, our findings are consistent with reports indicating a critical role of Rho in the regulation of $\mathrm{TJ}$ protein expression and TJ disassembly through activation of signaling pathways that regulate cytoskeletal organization.

Indeed, RhoA activation can reduce the endothelial barrier function via inactivation of MLC phosphatase (MLCP), leading to increased MLC phosphorylation and actin-myosin contractility (Itoh et al., 1999; Stamatovic et al., 2003). RhoA activation also results in the formation of stress fibers composed of filamentous actin and myosin II (Ridley and Hall, 1992; Wójciak-Stothard et al., 1998; Etienne-Manneville and Hall, 2002). Such mechanisms may contribute, at least in part, to an impaired intracellular movement of ZO-1 and contribute to increased levels of this TJ protein in the nuclear fractions of Tat-stimulated hCMEC/D3 
cells (Fig. 7). In support of this notion, it was demonstrated that cytochalasin B, which interferes with actin polymerization and contractile microfilaments, inhibited ZO-2 migration to the nuclei in MDCK monolayers (Islas et al., 2002). HIV-1 and Tat protein were shown to induce matrix metalloproteinase (MMP) and proteasome activities, which in turn can degrade TJ proteins, including $\mathrm{ZO}-1$ (Huang et al., 2009). Inhibition of both MMP and proteasome activity resulted in attenuation of HIV-1-induced altered expression of ZO-1 (Huang et al., 2009). Thus, nuclear translocation may protect this pool of ZO-1 against proteolytic degradation, whereas the membrane pool remains vulnerable to MMP and proteasome proteolysis. Because membrane ZO-1 is the major form of this TJ protein, its decrease results in diminished levels of total ZO-1 (Fig. 7).

In the present study, inhibition of Rho activity protected against Tat-induced alterations of the total and nuclear ZO-1 levels. In search for a possible target involved in such a protection, we focused on the role of CREB. The rationale for such studies was based on the literature reports indicating that CREB may act as an important trans-factor in regulation of TJ protein expression (Lui et al., 2007). Indeed, computational analysis revealed that ZO-1promoter contains several potential CREB binding sites (Chen et al., 2008). The role of CREB in ZO-1 expression was also confirmed by demonstrating that transcription factor JunD inhibited TJ protein ZO-1 transcription through binding to the CREB binding site at the ZO-1 promoter (Chen et al., 2008).

CREB is activated through Ser ${ }^{133}$ phosphorylation, and our data indicate that exposure to Tat both stimulates phosphorylation of CREB and activates its DNA binding activity (Fig. 3). These observations are in agreement with reports that Tat can activate CREB in various cell types, including monocytes and neuronal cells (Williams et al., 2005). Literature data suggest that Tat-induced activation of CREB may be mediated via calmodulin/ CaMKII-dependent p38 MAPK. In addition, intracellular Tat has been shown to phosphorylate CREB-1 through regulation of the ERK1/2 MAPK pathway (Gee et al., 2006, 2007). In the present study, we showed that Tat-mediated CREB activation was abolished by C3 adenovirus and ROCK inhibitor Y27632. Another potential mechanism of CREB phosphorylation may involve MLCP. In fact, we recently published that Tat-induced activation of RhoA can inhibit MLCP via phosphorylation of myosin phosphatase target subunit 1 and increased phosphorylation of MLC. Thus, phosphorylation of CREB appears to be placed directly downstream of RhoA. In support of this hypothTat plus control shRNA.
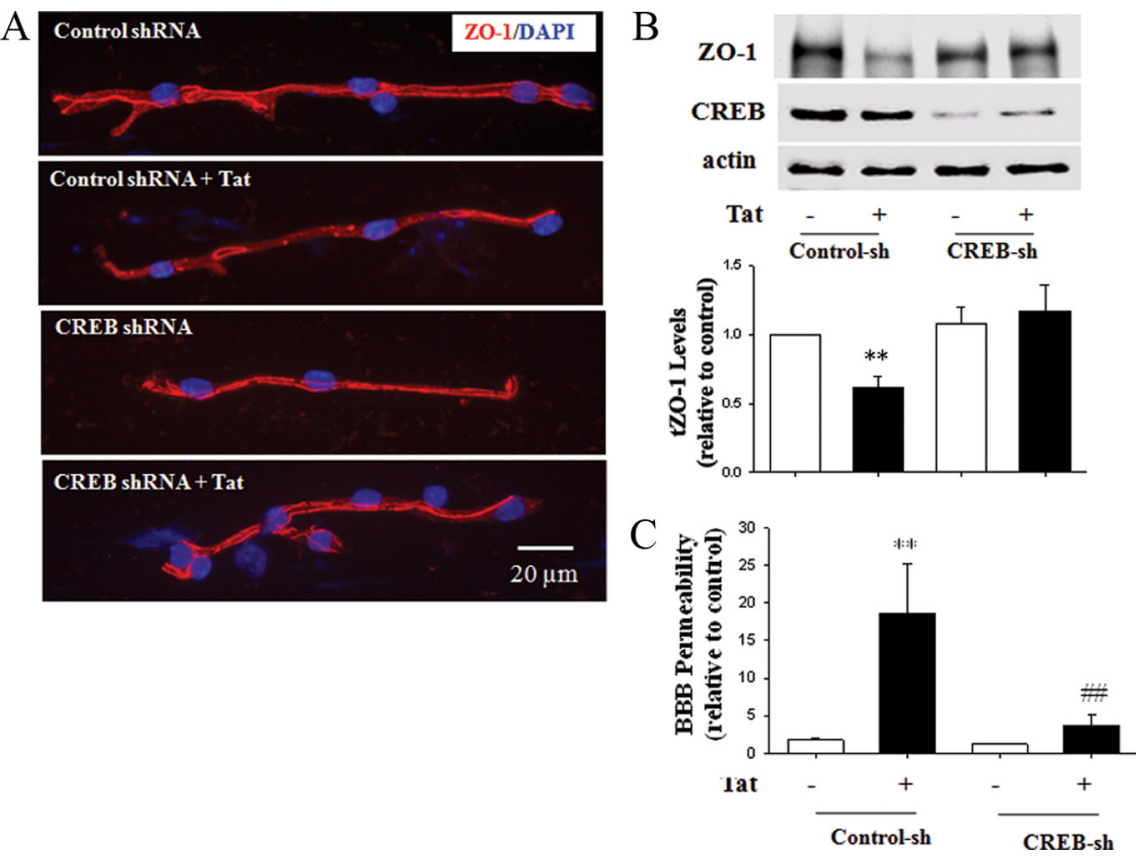

Figure 5. CREB modulates Tat-induced alteration of Z0-1 expression in vivo. Mice were injected with CREB shRNA (CREB-sh) or control shRNA (Control-sh) lentiviral particles into the ICA for $48 \mathrm{~h}$, followed by Tat injection ( $50 \mu \mathrm{g} /$ mouse). Brain microvessels were isolated from the ipsilateral hemisphere $24 \mathrm{~h}$ after Tat administration. $A$, CREB depletion protected against Tat-induced fragmentation of 20-1 immunoreactivity (red staining). DAPI staining was performed to visualize the nuclei (blue staining). $\boldsymbol{B}$, Immunoblotting analysis of Z0-1 and CREB expression. The blots are representative images from three to four mice and the quantified results are depicted in the form of bar graphs. Note also a marked decrease in CREB expression in microvessels of CREB shRNA-treated mice. C, CREB depletion protected against Tat-induced disruption of the BBB integrity. ${ }^{*} p<0.01$, compared to the respective control. ${ }^{\#} p<0.01$ data in the group Tat plus CREB shRNA lentiviral particles are significantly different compared with
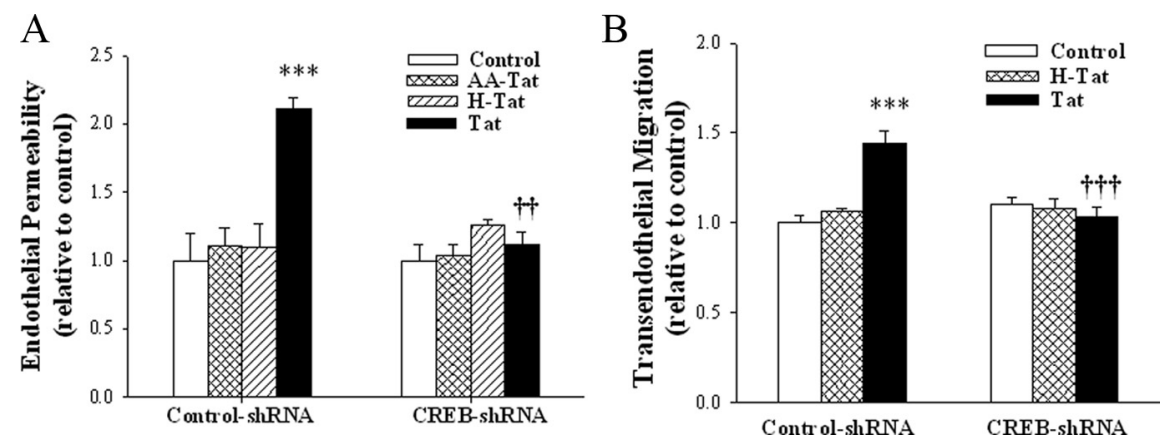

Figure 6. Depletion of CREB expression attenuates Tat-mediated increase in permeability and monocyte migration across endothelial monolayers. Cellular CREB was depleted as in Figure 3D, followed by treatment with Tat (100 nm), AA-Tat, or H-Tat (both negative controls) for $20 \mathrm{~h}$. A, Endothelial permeability was determined by measuring the flux of FITC- dextran of molecular weight $20 \mathrm{kDa}$ across hCMEC/D3 monolayers grown on $0.4 \mu \mathrm{m}$ membranes. $\boldsymbol{B}$, For monocyte transendothelial migration, $\mathrm{hC}$ MEC/D3 cells were grown on $3.0 \mu \mathrm{m}$ membrane inserts. Before treatment with Tat, $5 \times 10^{5}$ calcein AM-stained monocytic THP-1 cells were added on the top of confluent CREB-depleted and control shRNA-infected hCMEC/D3 monolayers for $4 \mathrm{~h}$ at $37^{\circ} \mathrm{C}$. Florescence was measured as a marker of monocytic cell migration. ${ }^{* *} p<0.001$, compared with the respective control (vehicletreated cells) at. ${ }^{+t} p<0.01$ or ${ }^{+t+} p<0.001$, data in cultures exposed to Tat plus (REB shRNA lentiviral particles are significantly different compared with Tat plus control shRNA.

esis, we demonstrated that CREB immunoprecipitates with MYLK (Fig. 3A) and inhibition of MYLK by ML-9 protected against Tat-induced phosphorylation of CREB (Fig. 3D).

To further evaluate the involvement of CREB in Tatmediated alterations of ZO-1 expression, CREB was depleted from hCMEC/D3 cells by infection with lentiviral particles containing CREB-specific shRNA. Unexpectedly, depletion of CREB prevented Tat-induced phosphorylation of MLC (Fig. $3 E$ ), a downstream kinase in the Rho signaling, indicating that 


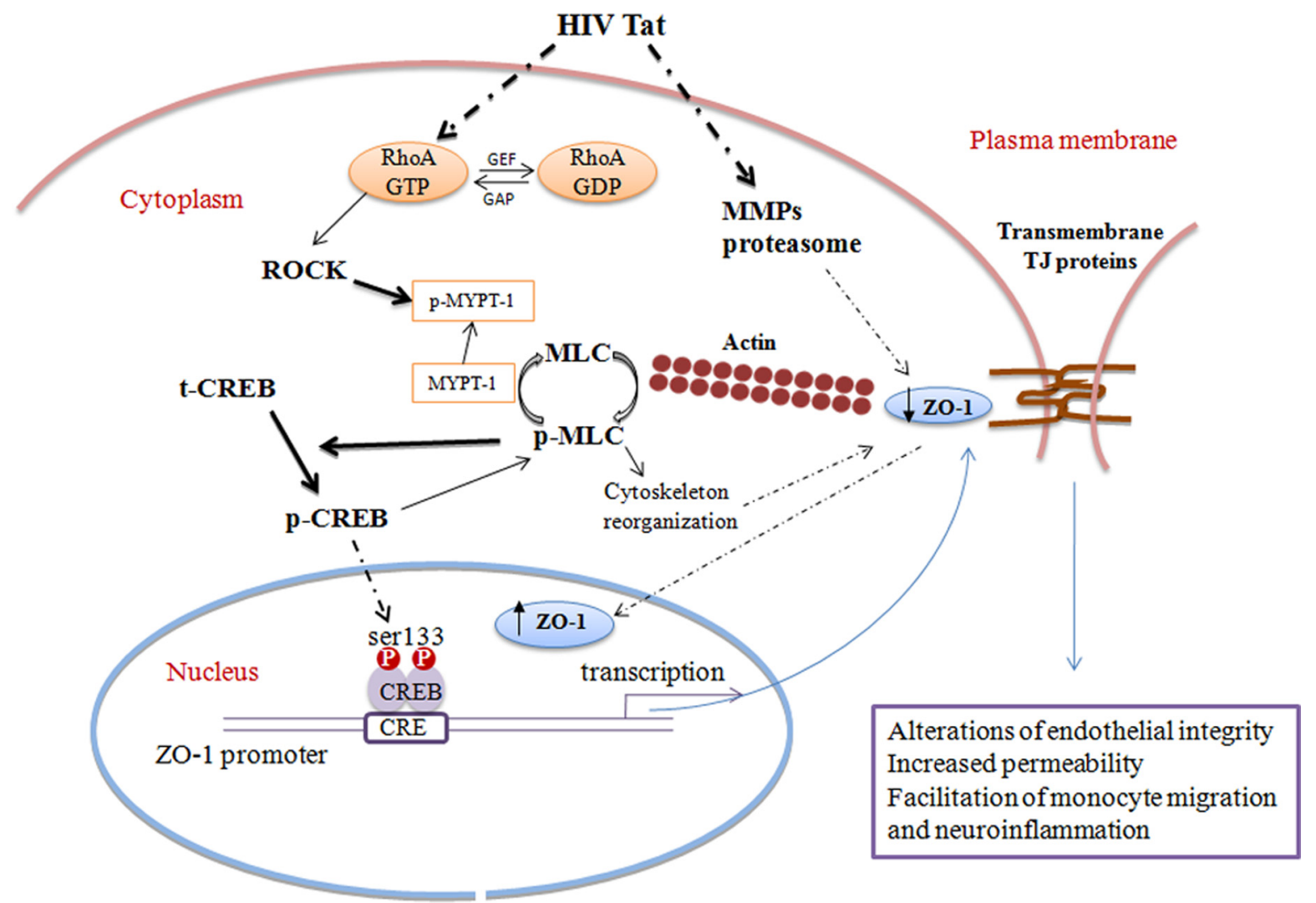

Figure 7. The proposed signaling pathway of Tat-induced nuclear localization of ZO-1 in human brain endothelial cells. HIV-1 protein Tat activates RhoA and downstream ROCK kinase, leading to phosphorylation of MLC, which then can mediate cross-linking F-actin into stress fibers and influence nuclear Z0-1 translocation. Stimulation of the Rho pathway results in phosphorylation of CREB, a transcriptional activator of ZO-1. In addition, exposure to HIV-1 or Tat protein stimulates MMPs and proteasome, which are responsible for degradation of TJ proteins, including ZO-1 (Huang et al., 2009). Nuclear translocation may protect this pool of Z0-1 against proteolytic degradation, whereas the membrane pool remains vulnerable to MMP and proteasome proteolysis. Because membrane ZO- 1 is the major form of this TJ protein, its decrease results in diminished levels of total ZO-1. In addition to stimulation of CREB phosphorylation by the Rho signaling, CREB also influences activation of MLC, indicating a close crosstalk between these two signaling pathways.

activation of downstream Rho mediators is dependent on CREB. Thus, our novel results suggest that activation of Rho and CREB are interrelated and regulate in concert ZO-1 expression in response to Tat treatment (Fig. 7). The role of CREB in Tat-mediated alterations of ZO-1 protein was also confirmed in vivo by depletion of this transcription factors from brain microvessels.

Although nuclear localization of TJ proteins has been recognized, its biological significance remains elusive. Nevertheless, evidence indicates that TJ proteins, such as ZO- 1 or ZO-2, have functions beyond regulation of endothelial permeability and are also involved in intracellular signaling and gene expression. For example, ZO proteins can associate with regulatory molecules (e.g., with adapter proteins, signaling molecules, or growth factors), thereby modulating the cell cycle. ZO proteins exhibit domains, such as several nuclear localization and nuclear export signals, enabling them to shuttle between the cytoplasm and the nucleus. There is no information available on the role of ZO-1 in the nuclear fraction; however, it might at least partially overlap with that of ZO-2. Literature reports indicate that the ratio between the amount of $\mathrm{ZO}-2$ localizing at $\mathrm{TJ}$ and the amount of nuclear $\mathrm{ZO}-2$ adds an additional variable in the control of epithelial cell proliferation and differentiation. Indeed, several studies have suggested that the nuclear localization of ZO-2 depends on the state of cellcell contacts in epithelial monolayers.

$\mathrm{ZO}-2$ was found to localize at the nucleus primarily in subconfluent epithelial cells before maturation of TJs but gradually exits the nucleus in a leptomycin-sensitive way when the monolayer reaches confluence. Nuclear accumulation of $\mathrm{ZO}-2$ is also believed to be a general response of epithelial and endothelial cells to environmental or mechanical stress because it can be induced by impairing cell-cell contacts by mechanical injury. Transient nuclear expression of ZO-2 has also been observed after heat shock and chemical insults in cerebral endothelial cells and kidney epithelial and endothelial cells (Islas et al., 2002; Traweger et al., 2003). Results of the present study demonstrate that CREB depletion prevents Tatinduced nuclear accumulation of ZO-1 and at the same time attenuates Tat-stimulated endothelial hyperpermeability and enhanced monocyte migration (Fig. 5). Thus, targeting of ZO-1 into the nuclei has profound effects on the functional status of brain endothelial cells.

In summary, the results of the present study demonstrate that exposure to HIV-1 protein Tat results in a decrease in total cellular levels of ZO-1 and an increase in nuclear levels of this TJ protein. Mechanistically, stimulation of Rho signaling and activation of CREB appear to be responsible for triggering nuclear accumulation of ZO-1. Preventing nuclear accumulation of ZO-1 by depletion of CREB attenuated Tat-induced dysfunction of endothelial barrier. Overall, these results indicate the importance of small GTPase, such as Rho, in regulation of TJ protein expression, localization, and modulation of the brain endothelial integrity in the context of HIV-1 brain infection. 


\section{References}

Adamson RH, Curry FE, Adamson G, Liu B, Jiang Y, Aktories K, Barth H, Daigeler A, Golenhofen N, Ness W, Drenckhahn D (2002) Rho and rho kinase modulation of barrier properties: cultured endothelial cells and intact microvessels of rats and mice. J Physiol 539:295-308.

András IE, Pu H, Tian J, Deli MA, Nath A, Hennig B, Toborek M (2005) Signaling mechanisms of HIV-1 Tat-induced alterations of claudin-5 expression in brain endothelial cells. J Cereb Blood Flow Metab 25:1159-1170.

Bauer H, Zweimueller-Mayer J, Steinbacher P, Lametschwandtner A, Bauer HC (2010) The dual role of zonula occludens (ZO) proteins. J Biomed Biotechnol 2010:402593.

Baumer Y, Burger S, Curry FE, Golenhofen N, Drenckhahn D, Waschke J (2008) Differential role of Rho GTPases in endothelial barrier regulation dependent on endothelial cell origin. Histochem Cell Biol 129:179-191.

Betanzos A, Huerta M, Lopez-Bayghen E, Azuara E, Amerena J, GonzálezMariscal L (2004) The tight junction protein ZO-2 associates with Jun, Fos and C/EBP transcription factors in epithelial cells. Exp Cell Res 292:51-66.

Boven LA, Middel J, Verhoef J, De Groot CJ, Nottet HS (2000) Monocyte infiltration is highly associated with loss of the tight junction protein zonula occludens in HIV-1-associated dementia. Neuropathol Appl Neurobiol 26:356-360.

Carbajal JM, Schaeffer RC Jr (1999) RhoA inactivation enhances endothelial barrier function. Am J Physiol 277:C955-C964.

Chen J, Xiao L, Rao JN, Zou T, Liu L, Bellavance E, Gorospe M, Wang JY (2008) JunD represses transcription and translation of the tight junction protein zona occludens-1 modulating intestinal epithelial barrier function. Mol Biol Cell 19:3701-3712.

Chen L, Swartz KR, Toborek M (2009) Vessel microport technique for applications in cerebrovascular research. J Neurosci Res 87:1718-1727.

Dallasta LM, Pisarov LA, Esplen JE, Werley JV, Moses AV, Nelson JA, Achim CL (1999) Blood-brain barrier tight junction disruption in human immunodeficiency virus-1 encephalitis. Am J Pathol 155:1915-1927.

De Marco A, Dans PD, Knezevich A, Maiuri P, Pantano S, Marcello A (2010) Subcellular localization of the interaction between the human immunodeficiency virus transactivator Tat and the nucleosome assembly protein 1. Amino Acids 38:1583-1593.

Etienne-Manneville S, Hall A (2002) Rho GTPases in cell biology. Nature 420:629-635

Eugenin EA, Osiecki K, Lopez L, Goldstein H, Calderon TM, Berman JW (2006) CCL2/monocyte chemoattractant protein-1 mediates enhanced transmigration of human immunodeficiency virus (HIV)-infected leukocytes across the blood-brain barrier: a potential mechanism of HIV-CNS invasion and NeuroAIDS. J Neurosci 26:1098-1106.

Gee K, Angel JB, Ma W, Mishra S, Gajanayaka N, Parato K, Kumar A (2006) Intracellular HIV-Tat expression induces IL-10 synthesis by the CREB-1 transcription factor through Ser ${ }^{133}$ phosphorylation and its regulation by the ERK1/2 MAPK in human monocytic cells. J Biol Chem 281:31647-31658.

Gee K, Angel JB, Mishra S, Blahoianu MA, Kumar A (2007) IL-10 regulation by HIV-Tat in primary human monocytic cells: involvement of calmodulin/calmodulin-dependent protein kinase-activated p38 MAPK and Sp-1 and CREB-1 transcription factors. J Immunol 178:798-807.

Hirai F, Nakayamada S, Okada Y, Saito K, Kurose H, Mogami A, Tanaka Y (2007) Small GTPase Rho signaling is involved in betal integrinmediated up-regulation of intercellular adhesion molecule 1 and receptor activator of nuclear factor kappaB ligand on osteoblasts and osteoclast maturation. Biochem Biophys Res Commun 356:279-285.

Hofmann WA (2009) Cell and molecular biology of nuclear actin. Int Rev Cell Mol Biol 273:219-263.

Huang W, Eum SY, András IE, Hennig B, Toborek M (2009) PPARalpha and PPARgamma attenuate HIV-induced dysregulation of tight junction proteins by modulations of matrix metalloproteinase and proteasome activities. FASEB J 23:1596-1606.

Islas S, Vega J, Ponce L, González-Mariscal L (2002) Nuclear localization of the tight junction protein ZO-2 in epithelial cells. Exp Cell Res 274:138-148.

Itoh K, Yoshioka K, Akedo H, Uehata M, Ishizaki T, Narumiya S (1999) An essential part for Rho-associated kinase in the transcellular invasion of tumor cells. Nat Med 5:221-225.

Luabeya MK, Dallasta LM, Achim CL, Pauza CD, Hamilton RL (2000) Blood-brain barrier disruption in simian immunodeficiency virus encephalitis. Neuropathol Appl Neurobiol 26:454-462.

Lui WY, Wong EW, Guan Y, Lee WM (2007) Dual transcriptional control of claudin-11 via an overlapping GATA/NF-Y motif: positive regulation through the interaction of GATA, NF-YA, and CREB and negative regulation through the interaction of Smad, HDAC1, and mSin3A. J Cell Physiol 211:638-648.

Ma M, Nath A (1997) Molecular determinants for cellular uptake of Tat protein of human immunodeficiency virus type 1 in brain cells. J Virol 71:2495-2499.

McNeil E, Capaldo CT, Macara IG (2006) Zonula occludens-1 function in the assembly of tight junctions in Madin-Darby canine kidney epithelial cells. Mol Biol Cell 17:1922-1932.

Pederson T (2008) As functional nuclear actin comes into view, is it globular, filamentous, or both? J Cell Biol 180:1061-1064.

Persidsky Y, Heilman D, Haorah J, Zelivyanskaya M, Persidsky R, Weber GA, Shimokawa H, Kaibuchi K, Ikezu T (2006) Rho-mediated regulation of tight junctions during monocyte migration across the blood-brain barrier in HIV-1 encephalitis (HIVE). Blood 107:4770-4780.

Ridley AJ, Hall A (1992) The small GTP-binding protein rho regulates the assembly of focal adhesions and actin stress fibers in response to growth factors. Cell 70:389-399.

Schneeberger EE, Lynch RD (2004) The tight junction: a multifunctional complex. Am J Physiol Cell Physiol 286:C1213-C1228.

Seelbach M, Chen L, Powell A, Choi YJ, Zhang B, Hennig B, Toborek M (2010) Polychlorinated biphenyls disrupt blood-brain barrier integrity and promote brain metastasis formation. Environ Health Perspect 118:479-484.

Stamatovic SM, Keep RF, Kunkel SL, Andjelkovic AV (2003) Potential role of MCP-1 in endothelial cell tight junction "opening": signaling via Rho and Rho kinase. J Cell Sci 116:4615-4628.

Traweger A, Fuchs R, Krizbai IA, Weiger TM, Bauer HC, Bauer H (2003) The tight junction protein $\mathrm{ZO}-2$ localizes to the nucleus and interacts with the heterogeneous nuclear ribonucleoprotein scaffold attachment factor-B. J Biol Chem 278:2692-2700.

Weksler BB, Subileau EA, Perrière N, Charneau P, Holloway K, Leveque M, Tricoire-Leignel H, Nicotra A, Bourdoulous S, Turowski P, Male DK, Roux F, Greenwood J, Romero IA, Couraud PO (2005) Blood-brain barrier-specific properties of a human adult brain endothelial cell line. FASEB J 19:1872-1874.

Williams CA, Mondal D, Agrawal KC (2005) The HIV-1 Tat protein enhances megakaryocytic commitment of K562 cells by facilitating CREB transcription factor coactivation by CBP. Exp Biol Med (Maywood) 230:872-884.

Wojciak-Stothard B, Ridley AJ (2002) Rho GTPases and the regulation of endothelial permeability. Vascul Pharmacol 39:187-199.

Wójciak-Stothard B, Entwistle A, Garg R, Ridley AJ (1998) Regulation of TNF-alpha-induced reorganization of the actin cytoskeleton and cell-cell junctions by Rho, Rac, and Cdc42 in human endothelial cells. J Cell Physiol 176:150-165.

Yezid H, Konate K, Debaisieux S, Bonhoure A, Beaumelle B (2009) Mechanism for HIV-1 Tat insertion into the endosome membrane. J Biol Chem 284:22736-22746.

Zhong Y, Smart EJ, Weksler B, Couraud PO, Hennig B, Toborek M (2008) Caveolin-1 regulates human immunodeficiency virus-1 Tat-induced alterations of tight junction protein expression via modulation of the Ras signaling. J Neurosci 28:7788-7796.

Zhong Y, Hennig B, Toborek M (2010) Intact lipid rafts regulate HIV-1 Tat protein-induced activation of the Rho signaling and upregulation of P-glycoprotein in brain endothelial cells. J Cereb Blood Flow Metab 30: 522-533. 